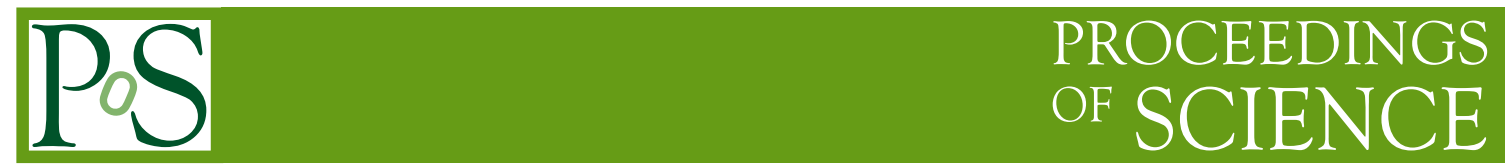

\title{
Transport and spectral functions in high-temperature QCD
}

\author{
Gert Aarts* \\ Department of Physics, Swansea University \\ Swansea, SA2 8PP, United Kingdom \\ E-mail: g.aarts@swan.ac.uk
}

The current status of transport coefficients in relativistic field theories at high temperature is reviewed. I contrast weak coupling results obtained using kinetic theory/diagrammatic techniques with strong coupling results obtained using gauge/gravity duality, and describe the recent developments in extracting transport coefficients and spectral functions from lattice QCD simulations. The fate of quarkonium at high temperature as seen from the lattice is briefly mentioned as well.

The XXV International Symposium on Lattice Field Theory

July 30-4 August 2007

Regensburg, Germany

\footnotetext{
* Speaker.
} 


\section{Introduction}

Transport coefficients characterize fluctuations and relaxation on long length and time scales in systems slightly away from thermal equilibrium. Consider a conserved current, $\partial_{t} n+\nabla \cdot \mathbf{j}=0$, in a system with a small nonuniformity in the density $n(t, \mathbf{x})$. To first approximation, a current will form to wash out the imbalance: $\mathbf{j}=-D \nabla n+\ldots$, where the dots indicate higher order terms in gradients. Combining the conservation law with this constitutive equation immediately yields the diffusion equation $\partial_{t} n=D \nabla^{2} n$. The proportionality factor $D$ is the diffusion constant and is the only quantity determined by the underlying microscopic theory: one may view it as a low-energy constant. Similarly, consider the conserved energy-momentum tensor $T^{\mu v}$. While in equilibrium $T^{i j}=\delta^{i j} P$, with $P$ the pressure, a perturbation characterized by a nonuniform flow field $u(t, \mathbf{x})$ will change this to (in the local rest frame, where $T^{0 i}=0$ )

$$
T^{i j}=\delta^{i j} P-\eta\left(\partial^{i} u^{j}+\partial^{j} u^{i}-\frac{2}{3} \delta^{i j} \partial_{l} u^{l}\right)-\zeta \delta^{i j} \partial_{l} u^{l}+\ldots
$$

Again the dots indicate higher order terms in gradients. The coefficient of the traceless combination is the shear viscosity $\eta$ and $\zeta$ is the bulk viscosity. Combining energy-momentum conservation with the constitutive equation above yields the hydrodynamic equations, predicting e.g. sound waves. In the context of thermal QCD one may therefore view hydrodynamics as the low-energy effective theory describing real-time dynamics at sufficiently large length and time scales. The form of the hydrodynamic equations is fixed by combining exact conservation laws and constitutive equations, which are obtained in a gradient expansion. In the latter, a number of low-energy constants appear, determined by QCD: shear viscosity $\eta$, bulk viscosity $\zeta$, electrical conductivity $\sigma$, diffusion constants $D$, etc. In this talk I review the progress in determining these coefficients from first principles.

The recent interest in transport in QCD and related theories is mainly due to the relativistic heavy ion program at RHIC [1]. The remarkable effectiveness of ideal hydrodynamics in describing heavy ion phenomenology [2, 3] suggests that transport coefficients are very small (when appropriately normalized). This in turns implies that thermalization times are short and interactions are strong, suggesting that in the temperature range $1 \lesssim T / T_{c} \lesssim 2$ the quark-gluon plasma is not a weakly coupled system of quarks and gluons, but instead strongly interacting (sQGP). A second reason for interest in transport is the fertile applicability of gauge/gravity duality (or AdS/CFT correspondence) to study strongly coupled thermal gauge theories in the hydrodynamic regime [4]. This has led to many (semi-)analytical results for those theories that admit a gravity dual and provides an important stimulus for QCD, where a gravity dual is not available. The best-known example concerns the ratio of the shear viscosity and the entropy density $s$,

$$
\frac{\eta}{s}=\frac{1}{4 \pi}
$$

which is obtained in all thermal gauge theories in the (strongly coupled) regime described by a dual gravity theory. This ratio is much smaller than in weakly coupled QCD, where

$$
\lim _{g \rightarrow 0} \frac{\eta}{s} \sim \frac{1}{g^{4} \ln 1 / g} \rightarrow \infty
$$

It is an open question what this ratio is in QCD just above the deconfinement transition. Below I describe recent progress in lattice QCD that will bring us closer to answering that question. 


\section{Transport coefficients at weak and strong coupling}

According to the Kubo relations, transport coefficients are given by the slope of current-current spectral functions, computed in thermal equilibrium, at vanishing energy. For example, the electrical conductivity $\sigma$ (or charge diffusion constant $D$ times susceptibility $\Xi$ ) is determined by

$$
\sigma=D \Xi=\lim _{\omega \rightarrow 0} \frac{\rho^{11}(\omega)}{2 \omega}
$$

where the current-current spectral function is given by

$$
\rho^{\mu v}(\omega)=\int d^{4} x e^{i \omega t}\left\langle\left[j^{\mu}(t, \mathbf{x}), j^{v}(0)\right]\right\rangle_{\mathrm{eq}} .
$$

For a single fermionic charge carrier with charge $e$ the electromagnetic current reads $j^{\mu}=e \bar{\psi} \gamma^{\mu} \psi$. Similarly, the shear and bulk viscosities are given by

$$
\eta=\lim _{\omega \rightarrow 0} \frac{\rho^{12,12}(\omega)}{2 \omega}, \quad \zeta=\frac{1}{9} \lim _{\omega \rightarrow 0} \frac{\rho^{i i, j j}(\omega)}{2 \omega},
$$

in terms of the energy-momentum tensor spectral function

$$
\rho^{\mu v, \rho \sigma}(\omega)=\int d^{4} x e^{i \omega t}\left\langle\left[T^{\mu v}(t, \mathbf{x}), T^{\rho \sigma}(0)\right]\right\rangle_{\mathrm{eq}} .
$$

\subsection{Weak coupling}

At weak coupling transport coefficients can be computed using either kinetic theory or by summing sets of Feynman diagrams. In this limit, transport coefficients are manifestly proportional to the mean free path or the inverse collisional width $1 / \Gamma$. They are therefore very large and inversely proportional to the coupling constants in the theory, see Eq. (1.3). In ultrarelativistic QCD the shear viscosity, electrical conductivity and diffusion constants have been computed to leadinglogarithm order [5] and subsequently to full leading order [6] in the gauge coupling. The relevant physics at leading-log are those $2 \leftrightarrow 2$ scattering processes that are logarithmically sensitive to infrared screening effects. The extension to full leading order requires, besides the other $2 \leftrightarrow 2$ scattering processes, also the inclusion of specific particle number changing processes [7]. The bulk viscosity is more complicated since particle number changing processes need to be included from the start; the first calculation in QCD can be found in Ref. [8]. It is shown that a parametrically correct estimate is given by

$$
\zeta \approx 15 \eta\left(1 / 3-v_{s}^{2}\right)^{2}
$$

where for light quarks the speed of sound $v_{s}$ is determined by $v_{s}^{2}-1 / 3 \sim \beta\left(g^{2}\right) \sim g^{4}$. The equivalence between kinetic theory and diagram summation in thermal gauge theories has been demonstrated in a number of papers $[9,10,11,12,13]$. A one-loop calculation is never sufficient; instead an infinite set of ladder diagrams has to be taken into account.

A complete leading-order calculation is also possible in the large $N_{f}$ limit, where $N_{f}$ indicates the number of flavours. This has been done using kinetic theory for massless quarks [14] and extended to massive quarks using diagrams [15]. Only Coulomb scattering processes contribute. Also in this limit one is effectively in the weakly coupled regime, since $\eta / s \sim N_{f} \rightarrow \infty$. 


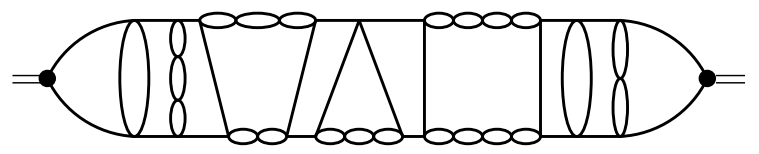

Figure 1: Typical skeleton diagram contributing to the shear viscosity in the $O(N)$ model at large $N$.

In all cases above only the leading-order result is known. This prevents any reasonable extrapolation to stronger coupling, unlike in the case of e.g. the pressure where the terms up to $g^{6} \ln 1 / g$ are known [16]. Recently the first subleading correction to a transport coefficient in a relativistic field theory has been computed by Moore [17], namely to the shear viscosity in scalar $\phi^{4}$ theory. The shear and bulk viscosity for single-component scalar theories have been computed a long time ago to leading order at weak coupling using ladder diagram summation [18]. In the $O(N)$ model the shear viscosity has also been found to leading order in the $1 / N$ expansion [19], using similar techniques as in large $N_{f}$ gauge theories. It is shown in Ref. [17] that the first subleading correction to the shear viscosity at weak coupling is sensitive to soft physics and can be extracted from the same set of diagrams that contribute at leading order by an expansion in terms of the thermal mass $m_{\text {th }} / T \sim \sqrt{\lambda}$. The result in the $O(N)$ model, with a $\lambda /(4 ! N)\left(\phi_{a} \phi_{a}\right)^{2}$ interaction, reads [17]

$$
\begin{aligned}
\eta & =\frac{T^{3}}{\lambda^{2}} \frac{3 N^{3}}{N+2}\left(3033.54+1548.3 \sqrt{\left(1+\frac{2}{N}\right) \frac{\lambda}{72}}+\mathscr{O}(\lambda)\right) \\
& =\frac{3 N^{2} T^{3}}{\lambda^{2}}\left(3033.54+1548.3 \sqrt{\frac{\lambda}{72}}+\mathscr{O}(\lambda, 1 / N)\right)
\end{aligned}
$$

where the second line is valid in the combined large $N$ and weak coupling limit. As mentioned above, the complete large $N$ result, without employing the weak-coupling expansion, is also known [19]. This includes a resummation of the thermal mass to all orders, as well as the inclusion of other diagrams suppressed in the weak coupling limit, see Fig. 1. Therefore, an assessment of the perturbative expansion can now be made. This is shown in Fig. 2, where the perturbative result

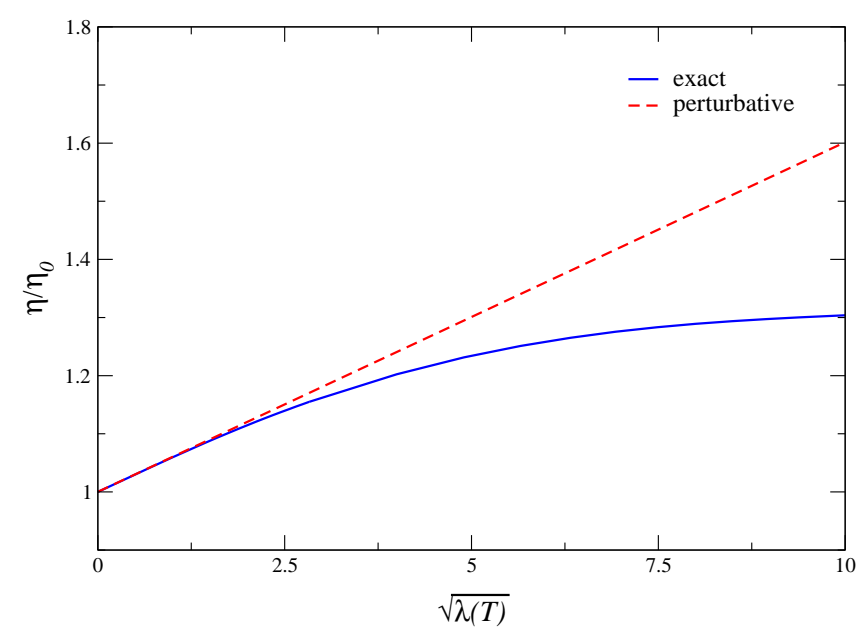

Figure 2: Shear viscosity $\eta$, normalized with the result at vanishing coupling, in the $O(N)$ model as a function of $\sqrt{\lambda}$ : comparison between the perturbative [17] and the exact [19] result in the large $N$ limit. 
$\eta / \eta_{0}=1+\# \sqrt{\lambda}$ is compared with the complete large $N$ result. Here $\eta_{0}$ is the result at vanishing coupling, which is used for normalization. From the first two terms, it appears that the convergence of the weak coupling expansion is not impressive. A possible approach to improve the convergence [17] would be to include the thermal mass without re-expanding, as has been tried for the pressure [20]. How to include all $\mathscr{O}(\lambda)$ effects is currently not known.

Considerable effort has been spent on nonrelativistic dynamics and diffusion of heavy quarks (with $M_{q} \gg T$ ). I will not discuss that here, but refer to Ref. [21] where definitions and a leadingorder calculation of the momentum diffusion coefficient can be found, and to Ref. [22] for the first next-to-leading order result at weak coupling.

\subsection{Strong coupling}

In order to complement weak coupling results as discussed above, it is desirable to have an analytic method tailored for strongly coupled field theories. For a certain class of thermal gauge theories such a method is available, via the gauge/gravity or AdS/CFT correspondence [23]. It uses the duality between a field theory at finite temperature and black holes in AdS space described in string theory. The best-known case relates $\mathscr{N}=4$ supersymmetric Yang-Mills theory to type IIB string theory on $\mathrm{AdS}_{5} \times S^{5}$, but there are many other field theories for which a gravity dual can be found (but not QCD). The parameters in field theory, the number of colours $N_{c}$ and the coupling constant $g^{2}$, are related to the parameters of string theory in AdS space. It turns out that the duality is most powerful when $g^{2}$ is small, since then loops in the string theory are suppressed, but with the 't Hooft coupling $\lambda=g^{2} N_{c}$ large, since then stringy effects are suppressed and the string theory reduces to a supergravity theory. Strongly coupled gauge theories, with large 't Hooft coupling, are therefore the natural area of application. Using the gauge/gravity correspondence, it has been shown that the field theories for which the duality holds behave hydrodynamically at large length and time scales, which supports the framework of (nearly ideal) hydrodynamics to understand the dynamics in thermal gauge theories at strong coupling.

The most famous example concerns the shear viscosity in $\mathscr{N}=4$ SYM. In the limit that both $N_{c}$ and $\lambda$ go to infinity, it is equal to [24]

$$
\eta=\frac{\pi}{8} N_{c}^{2} T^{3}
$$

In the same limit the entropy density is [25]

$$
s=\frac{\pi^{2}}{2} N_{c}^{2} T^{3}=\left.\frac{3}{4} S\right|_{\lambda=0},
$$

such that the ratio is $\eta / s=1 / 4 \pi$. This result for the ratio is universal and is achieved in all thermal gauge theories that can be described by a gravity dual $[26,27]$. The bulk viscosity vanishes due to conformal invariance.

The shear viscosity in $\mathscr{N}=4 \mathrm{SYM}$ at strong coupling is another rare example of a transport coefficient where the first subleading correction is known. One finds [28]

$$
\eta=\frac{\pi}{8} N_{c}^{2} T^{3}\left(1+\frac{75 \zeta(3)}{4 \lambda^{3 / 2}}+\ldots\right)
$$


The first correction to the ratio then reads

$$
\frac{\eta}{s}=\frac{1}{4 \pi}\left(1+\frac{135 \zeta(3)}{8 \lambda^{3 / 2}}+\ldots\right)
$$

It has been conjectured that $1 / 4 \pi$ is a lower bound for a wide class of systems, including e.g. the quark-gluon plasma and trapped atomic gases [29, 26]. For a recent critical assessment, see Ref. [30].

Other transport coefficients that have been computed include the $R$-charge diffusion coefficient and the bulk viscosity. These are not universal and depend on the theory under consideration. In $\mathscr{N}=4$ SYM the $R$-charge diffusion constant, susceptibility and conductivity are given by [31]

$$
D=\frac{1}{2 \pi T}, \quad \Xi=\frac{N_{c}^{2} T^{2}}{8}, \quad \sigma=\frac{N_{c}^{2} T}{16 \pi} .
$$

The bulk viscosity in a number of theories is discussed in Ref. [32]. For strongly coupled systems it is proposed that it satisfies (cf. Eq. (2.5) and the absent square)

$$
\zeta \geq 2 \eta\left(1 / 3-v_{s}^{2}\right)
$$

Also heavy quark dynamics has been studied extensively in strongly coupled $\mathscr{N}=4 \mathrm{SYM}$, see e.g. Ref. [33] for a clear discussion.

Due to the interest in $\mathscr{N}=4 \mathrm{SYM}$ as a testbed for thermal field dynamics, various quantities have also been studied at weak coupling. The ratio $\eta / s$ has been computed in this theory to full leading order in the small $\lambda$ limit (the result is independent of $N_{c}$ ) and compared with the same quantity in QCD [34]. Heavy quark diffusion has been studied to leading order at weak coupling in Ref. [35].

\section{Spectral functions}

So far, in discussing transport coefficients, the focus has been on the zero-energy limit of spectral functions. In this section I give a (restricted) review of spectral functions at arbitrary $\omega$, motivated mainly by hydrodynamic structure at weak and strong coupling. This will turn out to be useful for lattice QCD studies of spectral functions, to be discussed next.

In the weak coupling limit, current-current spectral functions have a characteristic energy dependence [36]. At large energy they increase as $\omega^{n}$, where the power $n$ is determined by the (mass) dimension. For example, the energy-momentum tensor spectral function (2.4) increases as $\omega^{4}$ and the EM current spectral function (2.2) as $\omega^{2}$ (unless there is a cancelation between components). At small energies, there is a transport peak. In free field theory, this peak manifests itself as a singular term,

$$
\frac{\rho(\omega)}{\omega} \sim 2 \pi \delta(\omega)
$$

reflecting that in a free theory the mean free path is infinite and transport coefficients diverge. Interactions regulate this singular behaviour and after the resummation of the collisional width $\Gamma$, the transport peak is modified to

$$
\frac{\rho(\omega)}{\omega} \sim \frac{2 \Gamma}{\omega^{2}+\Gamma^{2}}
$$




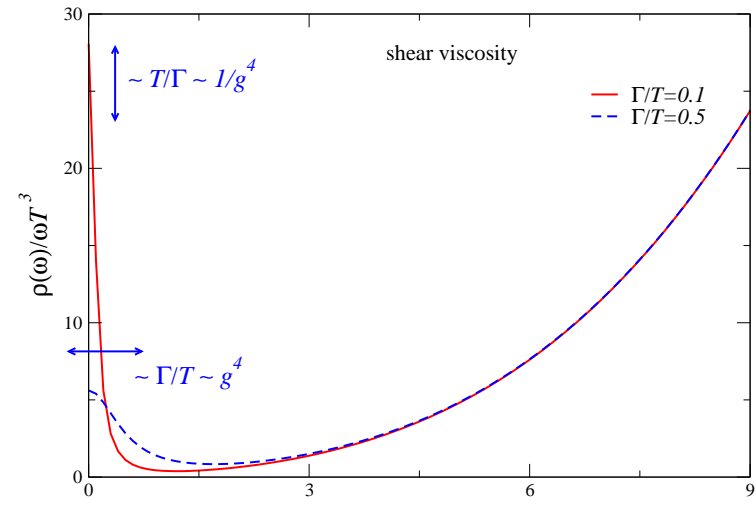

$\omega / T$

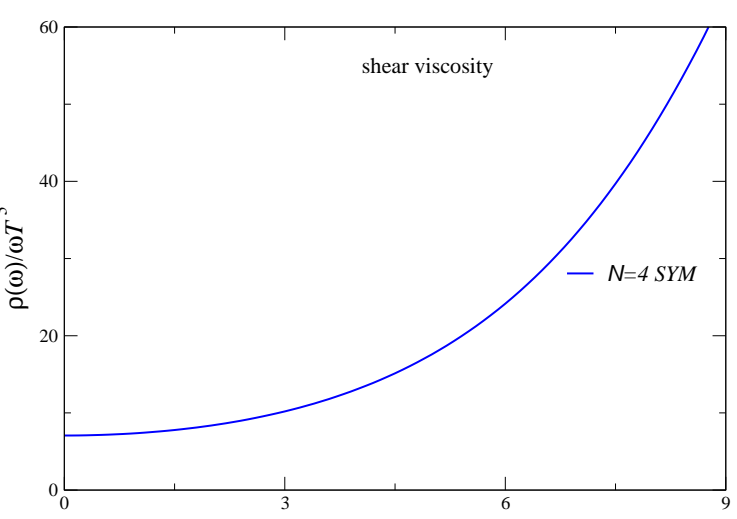

$\omega / T$

Figure 3: Shear viscosity: $\rho(\omega) / \omega T^{3}$ vs. $\omega / T$ in QCD (sketched) for two values of the collisional width $\Gamma / T=0.1,0.5$ (left) and in $\mathscr{N}=4 \mathrm{SYM}$ at strong coupling (right).

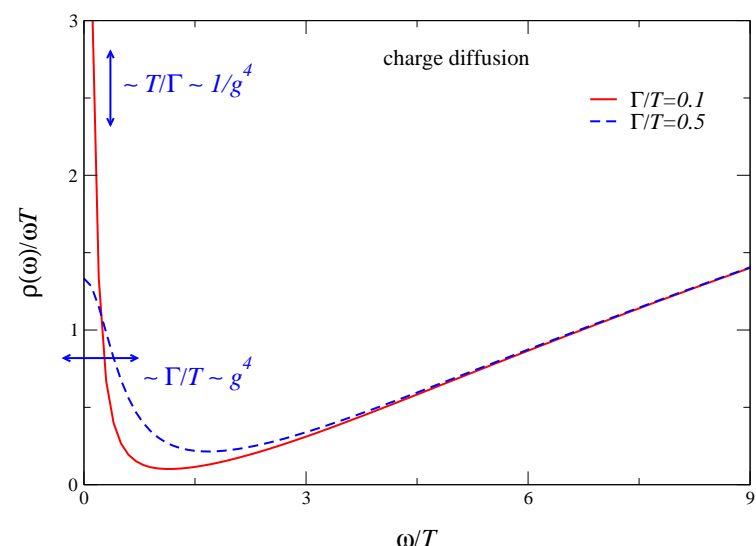

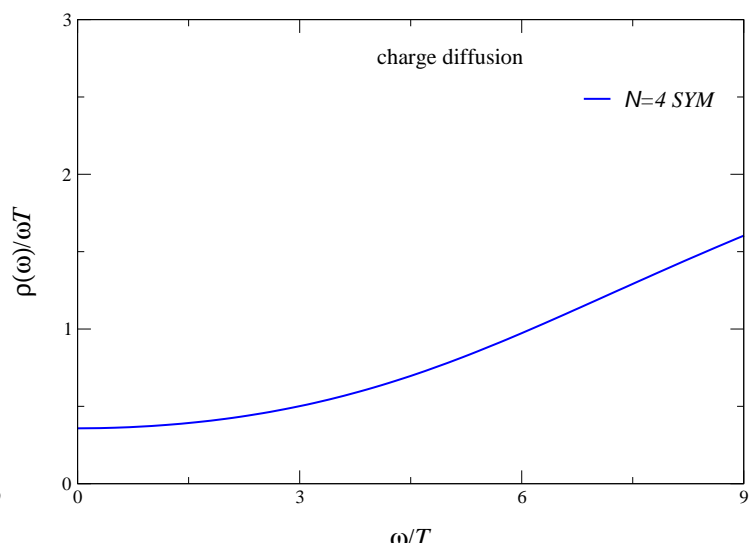

$\omega / T$

Figure 4: Charge diffusion: $\rho(\omega) / \omega T$ vs. $\omega / T$ in QCD (sketched) for two values of the collisional width $\Gamma / T=0.1,0.5$ (left) and in $\mathscr{N}=4 \mathrm{SYM}$ at strong coupling (right).

For simplicity, I parametrized the effect of interactions with a single constant width $\Gamma$; in reality the width depends on the momentum of the (quasi-)particles contributing to transport. At weak coupling the collisional width $\Gamma \sim g^{4} T$ : therefore the transport peak is narrow $\left(\sim g^{4}\right)$ and high $\left(\sim 1 / g^{4}\right)$.

The $\omega$ dependence of spectral functions of conserved charges, such as $\mathscr{E}=\int_{\mathbf{x}} T^{00}(t, \mathbf{x})$ or $Q=\int_{\mathbf{x}} j^{0}(t, \mathbf{x})$, is completely fixed by the conservation laws. For example, the spectral function for the total charge density reads

$$
\frac{\rho^{00}(\omega)}{\omega}=\Xi 2 \pi \delta(\omega)
$$

where $\Xi$ is again the susceptibility. This ensures that unequal-time correlation functions, such $\langle Q(t) Q(0)\rangle$, are in fact constant and equal to $V T \Xi$, where $V$ is the spatial volume and it is assumed that $\langle Q\rangle=0$. Here I only discuss spectral functions at zero spatial momentum; for nonzero spatial momentum see e.g. Ref. [37].

Combining the rising high-energy part and the transport peak at small energies yields spectral functions as shown in Fig. 3 (left) for the energy-momentum tensor (in the channel relevant for the 
shear viscosity) and Fig. 4 (left) for the EM current (charge diffusion). Here the width is taken as a free parameter and artificially increased to larger values $(\Gamma / T \lesssim 1)$, while keeping the rest of the spectral function unchanged. As a result the transport peak, which is narrow at weak coupling $(\Gamma / T \ll 1)$, becomes less singular and gets smeared out.

Interestingly enough, this behaviour can be compared with spectral functions computed in $\mathscr{N}=4 \mathrm{SYM}$ in the limit of large $N_{c}$ and large 't Hooft coupling $\lambda$, using gauge/gravity duality [38, 39]. The energy-momentum tensor spectral function has to be computed numerically by solving an ordinary differential equation, but for the $R$-current the analytical result is known and reads [40]

$$
\rho(\omega)=\frac{N_{c}^{2}}{16 \pi} \frac{\omega^{2} \sinh (\omega / 2 T)}{\cosh (\omega / 2 T)-\cos (\omega / 2 T)} .
$$

These spectral functions are shown in Figs. 3 and 4 (right). Rather than dividing by powers of $N_{c}, \mathrm{I}$ have simply put $N_{c}=3$. It is clear that at strong coupling the transport peak is no longer separated from the high-energy contribution and the spectral functions go smoothly to $\omega=0$. The intercept is of course proportional to the shear viscosity and the conductivity respectively.

Comparing the spectral functions on the left with those on the right makes it interesting to speculate what happens in QCD in the strong coupling regime above the deconfinement transition.

\section{Transport from lattice QCD}

We have seen that weak-coupling methods are probably not applicable in the interesting temperature regime of QCD probed by current heavy ion collisions. Furthermore, these calculations are so involved that in most cases only the leading-order result is currently known; it is an open question how to determine subleading corrections. On the other hand, the strong-coupling results discussed above are obtained in theories that are not QCD. So the important question is what can be said about transport in QCD when $1 \lesssim T / T_{c} \lesssim 3$, using nonperturbative lattice simulations.

The euclidean correlator calculated in numerical simulations is related to the corresponding spectral function via a dispersion relation,

$$
G_{E}\left(i \omega_{n}\right)=\int_{-\infty}^{\infty} \frac{d \omega}{2 \pi} \frac{\rho(\omega)}{\omega-i \omega_{n}},
$$

where $\omega_{n}=2 \pi n T(n \in \mathbb{Z})$ are the bosonic Matsubara frequencies. In euclidean time, with $0 \leq \tau<$ $1 / T$, this relation becomes

$$
G_{E}(\tau)=\int_{0}^{\infty} \frac{d \omega}{2 \pi} K(\omega, \tau) \rho(\omega)
$$

with the kernel

$$
K(\omega, \tau)=\frac{\cosh [\omega(\tau-1 / 2 T)]}{\sinh (\omega / 2 T)}=\left[1+n_{B}(\omega)\right] e^{-\omega \tau}+n_{B}(\omega) e^{\omega \tau}
$$

where $n_{B}(\omega)=1 /\left(e^{\omega / T}-1\right)$ is the Bose distribution. The first expression for the kernel shows the characteristic euclidean time dependence, while the second expression emphasizes that the correlator and its spectral function are essentially related via a Laplace transform, made periodic to satisfy the Kubo-Martin-Schwinger (periodicity) condition. 
If the euclidean correlator is known analytically, the spectral function can simply be obtained by analytic continuation,

$$
\rho(\omega)=2 \operatorname{Im} G_{E}\left(i \omega_{n} \rightarrow \omega+i \varepsilon\right) .
$$

However, if $G_{E}(\tau)$ is determined numerically on a lattice with $N_{\tau}=1 / a T$ points in the euclidean time direction ( $a$ is the temporal lattice spacing), Eq. (4.1) has to be inverted by some other means. This is an ill-posed inversion problem, since $G_{E}(\tau)$ is known at, say, $\mathscr{O}(10)$ data points, whereas $\rho(\omega)$ is needed at $\mathscr{O}\left(10^{3}\right)$ values (after imposing a high-energy cutoff $\omega_{\max }$ and discretizing the resulting finite interval $\left.0<\omega<\omega_{\max }\right)$.

One possible solution is to provide an Ansatz for the spectral function, with a small number of free parameters. In this case it is important to be able to judge the applicability of the Ansatz. An orthogonal approach is to avoid giving functional forms but only supply a minimal amount of prior information, such as positivity $(\omega \rho(\omega) \geq 0)$ and asymptotic behaviour $\left(\rho(\omega) \sim \omega^{n}\right.$ for large $\left.\omega\right)$. Methods based on this approach are usually collectively referred to as Bayesian techniques.

For a weakly coupled quark-gluon plasma, the extraction of transport coefficients is notoriously difficult, since euclidean correlators are remarkably insensitive to the structure of spectral functions at energies $\omega \ll T[36,41]$. However, at stronger coupling the transport peak is much broader and the small $\omega$ limit is no longer singular. As discussed above, smooth spectral functions are also found in $\mathscr{N}=4 \mathrm{SYM}$ at strong coupling. This opens up the possibility that transport coefficients are accessible in lattice QCD above the deconfinement transition.

So far the number of papers in the literature that have attempted to extract transport coefficients from the lattice in a head-on approach is very small. A first attempt to measure transport coefficients can be found in the pioneering paper by Karsch and Wyld, using an Ansatz [42]. This method was followed by Nakamura and Sakai for the shear and bulk viscosity [43]. For a critical discussion of the Ansatz, see Ref. [36]. S. Gupta used Bayesian methods to isolate the transport contribution at small energies in the case of the electrical conductivity [44]. In the past six months, two significant steps have been made. A standard approach to perform the analytical continuation using Bayesian techniques is known as the Maximum Entropy Method (MEM), to be discussed below. It was known from previous work that MEM performs poorly at small energies. Aarts, Allton, Foley, Hands and Kim have identified and resolved a numerical instability in MEM in the limit that $\omega \rightarrow 0$ and applied the new formulation to obtain the electrical conductivity [45]. Precisely determined correlators are essential to have control over the analytic continuation; while for the electrical conductivity this is not a problem, for the shear and bulk viscosity standard measurement techniques are insufficient. Meyer has applied a two-level algorithm to better determine energy-momentum correlators and found a result for the shear viscosity [46]. All calculations to date have been performed in quenched QCD, so one may think of the electrical conductivity in pure gauge theory as representing the transport properties of a single electrically charged quark diffusing through a gluon plasma.

In the following I describe in some detail the Maximum Entropy Method and indicate why the standard algorithm is unstable in the small $\omega$ region [45]. In MEM one reconstructs the most probable spectral function by extremizing the probability distribution $P[\rho \mid G H]$, i.e. the probability to find $\rho$, given the correlator $G$ and prior information $H$. Using an identity for conditional probabilities, $P[\rho G \mid H]=P[\rho \mid G H] P[G \mid H]=P[G \mid \rho H] P[\rho \mid H], P[\rho \mid G H]$ is written as the product of a 

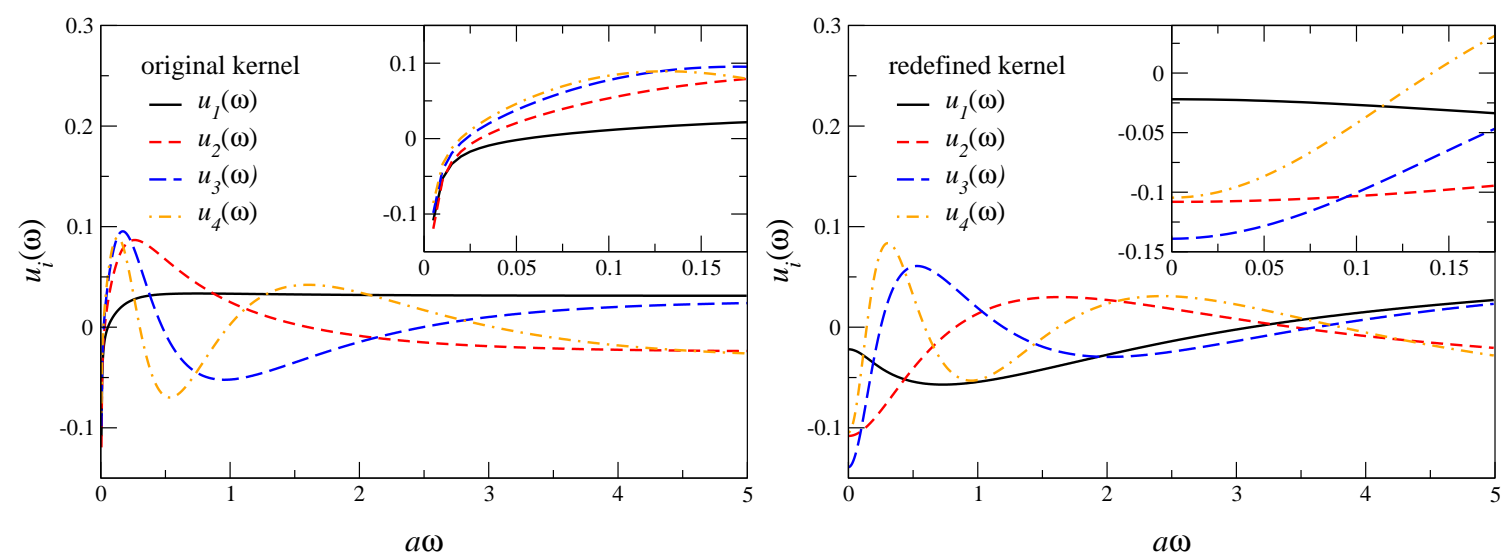

Figure 5: First four basis functions $u_{i}(\omega)$ in the singular value decomposition as a function of $a \omega$ for $a \omega_{\max }=5, N_{\omega}=1000, N_{\tau}=24$, using the standard (left) and the redefined kernel (right). The inset shows a blow-up of the small energy region.

standard likelihood function, $P[G \mid \rho H] \sim e^{-L}$ ( $\chi^{2}$ fit), and a prior probability, $P[\rho \mid H] \sim e^{\alpha S}$ which is independent of the data. $P[G \mid H]$ is a normalization factor. The prior information is encoded in the entropy term,

$$
S=\int \frac{d \omega}{2 \pi}\left[\rho(\omega)-m(\omega)-\rho(\omega) \log \frac{\rho(\omega)}{m(\omega)}\right]
$$

via the default model $m(\omega)$. Therefore, the combined function to extremize is $P[\rho \mid G H] \sim e^{-L+\alpha S}$, where $\alpha$ determines the relative weight of the data versus the prior information [47].

The most important aspect for my purpose here is the reduction step. Recall that after discretization $\rho(\omega)$ is wanted at $N_{\omega}=\mathscr{O}\left(10^{3}\right)$ values whereas the correlator is only known at $\mathscr{O}(10)$ points. I denote with $N$ the number of time slices included in the analysis; due to reflection symmetry, $N \leq N_{\tau} / 2$. To make this well-defined, the number of coefficients parametrizing the spectral function cannot exceed $N$, or in other words, $\rho(\omega)$ has to be restricted to an (at most) $N$ dimensional subspace. In Bryan's algorithm [48] this is achieved via a singular value decomposition (SVD) of the kernel $K\left(\omega_{n}, \tau_{i}\right)$. Viewed as an $N_{\omega} \times N$ matrix, the kernel is written as $K=U W V^{T}$, where $U$ is an $N_{\omega} \times N$ matrix, with $U^{T} U=\mathbb{1}_{N \times N}, W$ is a diagonal $N \times N$ matrix, and $V$ is an orthogonal $N \times N$ matrix. The $N$ dimensional subspace is spanned by the column vectors of $U$ : $u_{i}\left(\omega_{n}\right)=U_{n i}$. These basis vectors are orthogonal but not complete. An analysis of the extremum conditions shows that it is natural to write the spectral function in terms of these basis vectors as $\rho(\omega)=m(\omega) \exp \sum_{i=1}^{N} c_{i} u_{i}(\omega)$. This ensures positivity and provides the actual reduction step. Extremizing the probability distribution leads to nonlinear equations for the $N$ coefficients $c_{i}$.

The first four basis functions in the SVD are shown in Fig. 5 (left), for a typical choice of $N=N_{\tau} / 2, N_{\omega}$, and $\omega_{\max }$. A blow-up of the small energy region reveals that the basis functions appear to diverge when $\omega \rightarrow 0$, although they are normalized (the smallest energy included here is $\left.a \Delta \omega=a \omega_{\max } / N_{\omega}=0.005\right)$. This apparent divergence is due to the singular behaviour of the kernel (4.3): in the limit that $\omega \rightarrow 0$ one finds that $K(\omega, \tau)=2 T / \omega+\mathscr{O}(\omega / T)$. Note that the leading singular term is $\tau$ independent; all $\tau$ independence resides in the subleading terms. The behaviour of the basis functions is therefore indicative of a real problem, which cannot be solved by e.g. decreasing $\Delta \omega$. In actual applications of MEM, we (and others) found irregular behaviour at small 


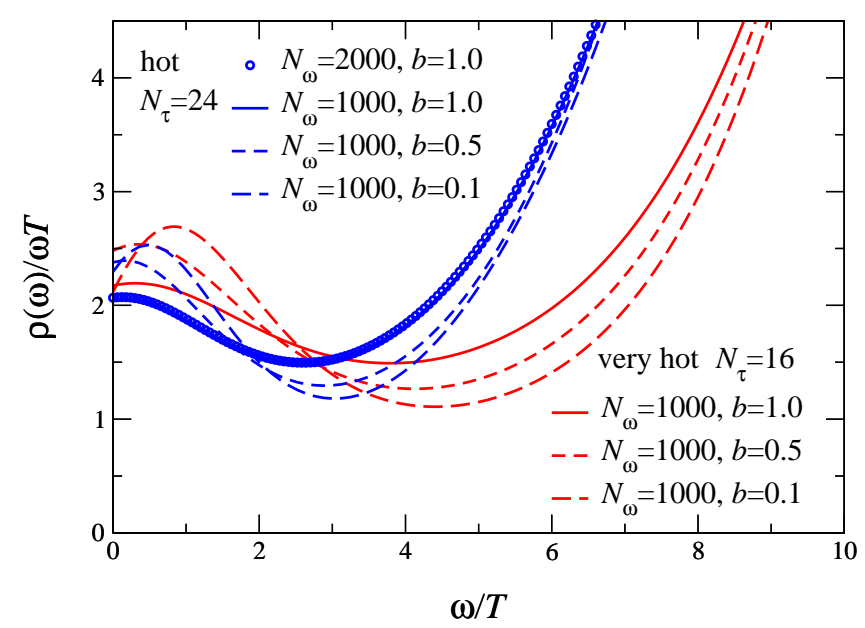

Figure 6: Current-current spectral function $\rho(\omega) / \omega T$, where $j^{i}=\bar{\psi} \gamma^{i} \psi$, as a function of $\omega / T$ in quenched QCD for $T / T_{c} \sim 1.5$ (hot, $N_{\tau}=24$ ) and 2.25 (very hot, $N_{\tau}=16$ ). The intercept at $\omega=0$ is proportional to the electrical conductivity. Results are shown for $N_{\omega}=1000,2000$ and $b=1.0,0.5,0.1$ at fixed $a \omega_{\max }=5$.

$\omega$, in particular at the smallest nonzero value of $\omega$. Needless to say that this prevents access to the transport properties encoded in euclidean correlators. It is worth pointing out that this problem only appears at finite temperature, since at zero temperature the kernel reduces to $K(\omega, \tau)=e^{-\omega \tau}$ and the limit $\omega \rightarrow 0$ is smooth. It is a manifestation of the fact that the limits $\omega \rightarrow 0$ and $T \rightarrow 0$ do not commute, which is well-known in thermal field theory.

Once the problem is identified, it is straightforward to solve it. The $1 / \omega$ divergence can be avoided by defining

$$
\bar{K}(\omega, \tau)=\frac{\omega}{2 T} K(\omega, \tau), \quad \bar{\rho}(\omega)=\frac{2 T}{\omega} \rho(\omega) .
$$

Since $K(\omega, \tau) \rho(\omega)=\bar{K}(\omega, \tau) \bar{\rho}(\omega)$ the standard relation with the euclidean correlator holds. However, the modified kernel is finite when $\omega \rightarrow 0: \bar{K}(0, \tau)=1$. A SVD of $\bar{K}$ yields new basis functions $\bar{u}_{i}(\omega)$. The first four are shown in Fig. 5 (right). Clearly they take a finite value when $\omega \rightarrow 0$, and the $\omega=0$ point can be included in the analysis. The redefined spectral function is expanded as $\bar{\rho}(\omega)=\bar{m}(\omega) \exp \sum_{i=1}^{N} \bar{c}_{i} \bar{u}_{i}(\omega)$ and the same MEM routine can be used to find the coefficients $\bar{c}_{i}$. MEM now reconstructs $\bar{\rho} \sim \rho / \omega$ rather than $\rho$. This reshuffling of powers of $\omega$ is nontrivial, since $\bar{\rho}$ and $\rho$ are not expanded in a complete set: the reduction step restricts $\bar{\rho}$ to a different subspace with manifestly different properties, in particular in the small $\omega$ limit.

A second (minor) modification is needed to access $\rho(\omega) / \omega$ at zero $\omega$, relevant for transport coefficients. For spectral functions of fermion bilinears, such as $j^{\mu}=\bar{\psi} \gamma^{\mu} \psi$, the traditional default model is $\bar{m}(\omega) \sim m(\omega) / \omega \sim \omega$, determined by the high-energy behaviour $\rho(\omega) \sim \omega^{2}$. Unfortunately, this introduces a bias and puts the intercept equal to zero from the start. To avoid this, one may use $\bar{m}(\omega) \sim(b+\omega)$, where $b>0$ is a parameter that can be used to assess default model dependence at small $\omega$.

We have applied the modified algorithm to the problem of the electrical conductivity (or charge diffusion) in quenched QCD with light staggered fermions and performed simulations on a fine lattice at $\beta=7.192$ of size $64^{3} \times N_{\tau}$ above the deconfinement transition. The quarks are so light that 
chiral symmetry restoration is clearly visible when comparing pseudoscalar and scalar correlators [49]. In Fig. 6, the spectral function normalized by $\omega T$ is shown for not too large $\omega$ at two temperatures above the deconfinement transition: $T / T_{c} \sim 1.5\left(N_{\tau}=24\right)$ and $T / T_{c} \sim 2.25\left(N_{\tau}=16\right)$. In both cases results for three values of the default model parameter $b$ are shown. The slight spread of the curves gives an indication of the uncertainty in the MEM reconstruction. There is no dependence on the discretization along the $\omega$ axis, as can be seen from the results at $N_{\tau}=24$ using $N_{\omega}=1000$ and 2000 at fixed $\omega_{\max }$. From the intercept the conductivity is found to be $\sigma / T=0.4 \pm 0.1$ with no significant temperature dependence. This result is normalized to a single flavour and should be multiplied with the sum of the electric charge squared for light flavours. The error is systematic and due to the MEM uncertainty. The statistical error is expected to be smaller. This result is indicative of strong interactions: at weak coupling the conductivity behaves as $\sigma / T \sim 1 /\left(g^{4} \ln 1 / g\right) \rightarrow \infty$, whereas at strong coupling the scale is set solely by the temperature.

A similar conclusion has been drawn by Meyer in the case of the shear viscosity. In Ref. [46] an upper bound $\eta / s \lesssim 1$ is obtained in $\mathrm{SU}(3)$ gauge theory on lattices at $T / T_{c}=1.24$ ( $\beta=6.2$, $\left.20^{3} \times 8\right)$ and $1.65\left(\beta=6.408,28^{3} \times 8\right)$.

\section{Quarkonium at high temperature}

Another signal of strong interactions in the quark-gluon plasma is the survival of charmonium and other heavy quark mesons above $T_{c}$. This has been studied on the lattice a few years ago using the Maximal Entropy Method [50, 51, 52]. A more recent extensive study of charmonium and bottonium spectral functions can be found in Ref. [53]. This topic has been discussed last year in Hatsuda's plenary talk [54], so I briefly mention developments that took place recently and were discussed at this Conference.

Up to last year, all studies were performed in quenched QCD. The TrinLat collaboration has carried out dynamical simulations with two flavours on highly anisotropic lattices, which can be used to study charmonium at zero and nonzero temperature [55]. A spectral function analysis above $T_{c}$, using the modified version of MEM described in the previous section, can be found in Ref. [56]. The results suggest that the S-waves $\left(J / \psi\right.$ and $\left.\eta_{c}\right)$ survive up to temperatures close to $2 T_{c}$, while the P-waves $\left(\chi_{c 0}\right.$ and $\left.\chi_{c 1}\right)$ melt away below $1.2 T_{c}$. However, there are systematic uncertainties that need to be improved in order to make these conclusions more firm, in particular simulations at a finer lattice spacing would be desirable. One reason this is necessary is to better understand the appearance of artefacts at larger energy introduced by the finite lattice spacing, which have been discussed for a variety of lattice fermion formulations (Wilson, staggered, domain wall, overlap) in the free field limit $[57,37,58]$.

The presence of the transport contribution at small $\omega$ can interfere with spectral features at larger $\omega$ when not properly disentangled, as emphasized by Umeda [59]. This can be partially avoided by subtracting the midpoint value at $\tau=1 / 2 T$ from the correlator, which has the effect of suppressing the contribution at small $\omega$. A spectral analysis can then be done on $G(\tau)-G(1 / 2 T)$.

Petreczky and Mócsy have provided a closer look at potential models, traditionally used to study quarkonium at zero temperature. Doubts whether potential models can describe quarkonium correlators at finite temperature have been expressed in Ref. [60]. A lower melting temperature than 
usually obtained with MEM is found when a potential model based on lattice QCD simulations is used, both in the quenched approximation [61] as well as in the theory with $2+1$ flavours [62].

In order to avoid conceptual problems with the extension of zero-temperature potential models to nonzero temperature, a real-time static potential, firmly based in thermal field theory, was introduced in Ref. [63]. When applied to quarkonium [64], the results seem to support the standard interpretation of results obtained with MEM.

\section{Summary}

Results from relativistic heavy ion collisions at RHIC have highlighted the importance of understanding transport and hydrodynamical behaviour in QCD above the deconfined transition. Nonperturbative first-principle calculations of spectral functions, especially at small energies $\omega \lesssim$ $T$, are badly needed. Since this involves inherently real-time physics, it is a difficult problem for lattice QCD, but recently several steps forward have been made. Using multi-level algorithms, accurately determined euclidean correlators of the energy-momentum tensor are now available. Concerning the analytical continuation to real time, an instability at small energies in the standard Maximum Entropy Method, preventing access to hydrodynamical features of spectral functions, has been found and resolved. The first results support the idea that the quark-gluon plasma is strongly interacting in the temperature range $1 \lesssim T / T_{c} \lesssim 2.5$. Extension of the work described here will hopefully yield a better understanding of the hydrodynamical regime of thermal QCD from first principles.

\section{Acknowledgments}

It is a great pleasure to thank my collaborators Chris Allton, Justin Foley, Simon Hands and Seyong Kim, Bugra Oktay, Mike Peardon and Jonivar Skullerud, and Jose Martínez Resco. I also thank Carlos Nunez for discussion. This work is supported by a PPARC Advanced Fellowship.

\section{References}

[1] I. Arsene et al. [BRAHMS Collaboration], Nucl. Phys. A 757 (2005) 1 [nucl-ex/0410020]; B. B. Back et al., ibid. 28 [nucl-ex/0410022]; J. Adams et al. [STAR Collaboration], ibid. 102 [nucl-ex/0501009]; K. Adcox et al. [PHENIX Collaboration], ibid. 184 [nucl-ex/0410003].

[2] P. F. Kolb, P. Huovinen, U. W. Heinz and H. Heiselberg, Phys. Lett. B 500 (2001) 232 [hep-ph/0012137]; P. F. Kolb and U. W. Heinz, nucl-th/0305084.

[3] D. Teaney, J. Lauret and E. V. Shuryak, Phys. Rev. Lett. 86 (2001) 4783 [nucl-th/0011058]; nucl-th/0110037.

[4] D. T. Son and A. O. Starinets, arXiv:0704.0240 [hep-th].

[5] P. Arnold, G. D. Moore and L. G. Yaffe, JHEP 0011 (2000) 001 [hep-ph/0010177].

[6] P. Arnold, G. D. Moore and L. G. Yaffe, JHEP 0305 (2003) 051 [hep-ph/0302165].

[7] P. Arnold, G. D. Moore and L. G. Yaffe, JHEP 0301 (2003) 030 [hep-ph/0209353].

[8] P. Arnold, C. Dogan and G. D. Moore, Phys. Rev. D 74 (2006) 085021 [hep-ph/0608012]. 
[9] M. A. Valle Basagoiti, Phys. Rev. D 66 (2002) 045005 [hep-ph/0204334].

[10] G. Aarts and J. M. Martínez Resco, JHEP 0211 (2002) 022 [hep-ph/0209048].

[11] G. Aarts and J. M. Martínez Resco, Phys. Rev. D 68 (2003) 085009 [hep-ph/0303216].

[12] J. S. Gagnon and S. Jeon, Phys. Rev. D 75 (2007) 025014 [hep-ph/0610235]; arXiv:0708.1631 [hep-ph].

[13] M. E. Carrington and E. Kovalchuk, arXiv:0709.0706 [hep-ph].

[14] G. D. Moore, JHEP 0105 (2001) 039 [hep-ph/0104121].

[15] G. Aarts and J. M. Martínez Resco, JHEP 0503 (2005) 074 [hep-ph/0503161].

[16] K. Kajantie, M. Laine, K. Rummukainen and Y. Schroder, Phys. Rev. D 67 (2003) 105008 [hep-ph/0211321].

[17] G. D. Moore, arXiv:0706.3692 [hep-ph], Phys. Rev. D (to appear).

[18] S. Jeon, Phys. Rev. D 52 (1995) 3591 [hep-ph/9409250].

[19] G. Aarts and J. M. Martínez Resco, JHEP 0402 (2004) 061 [hep-ph/0402192].

[20] F. Karsch, A. Patkos and P. Petreczky, Phys. Lett. B 401 (1997) 69 [hep-ph/9702376].

[21] G. D. Moore and D. Teaney, Phys. Rev. C 71 (2005) 064904 [hep-ph/0412346].

[22] S. Caron-Huot and G. D. Moore, arXiv:0708.4232 [hep-ph].

[23] J. M. Maldacena, Adv. Theor. Math. Phys. 2 (1998) 231 [Int. J. Theor. Phys. 38 (1999) 1113] [hep-th/9711200].

[24] G. Policastro, D. T. Son and A. O. Starinets, Phys. Rev. Lett. 87 (2001) 081601 [hep-th/0104066].

[25] S. S. Gubser, I. R. Klebanov and A. W. Peet, Phys. Rev. D 54 (1996) 3915 [hep-th/9602135].

[26] P. Kovtun, D. T. Son and A. O. Starinets, Phys. Rev. Lett. 94 (2005) 111601 [hep-th/0405231].

[27] A. Buchel and J. T. Liu, Phys. Rev. Lett. 93 (2004) 090602 [hep-th/0311175].

[28] A. Buchel, J. T. Liu and A. O. Starinets, Nucl. Phys. B 707 (2005) 56 [hep-th/0406264].

[29] P. Kovtun, D. T. Son and A. O. Starinets, JHEP 0310 (2003) 064 [hep-th/0309213].

[30] A. Cherman, T. D. Cohen and P. M. Hohler, arXiv:0708.4201 [hep-th].

[31] G. Policastro, D. T. Son and A. O. Starinets, JHEP 0209 (2002) 043 [hep-th/0205052].

[32] A. Buchel, arXiv:0708.3459 [hep-th].

[33] C. P. Herzog, A. Karch, P. Kovtun, C. Kozcaz and L. G. Yaffe, JHEP 0607 (2006) 013 [hep-th/0605158].

[34] S. C. Huot, S. Jeon and G. D. Moore, Phys. Rev. Lett. 98 (2007) 172303 [hep-ph/0608062].

[35] P. M. Chesler and A. Vuorinen, JHEP 0611 (2006) 037 [hep-ph/0607148].

[36] G. Aarts and J. M. Martínez Resco, JHEP 0204 (2002) 053 [hep-ph/0203177]; Nucl. Phys. Proc. Suppl. 119 (2003) 505 [hep-lat/0209033].

[37] G. Aarts and J. M. Martínez Resco, Nucl. Phys. B 726 (2005) 93 [hep-lat/0507004].

[38] D. Teaney, Phys. Rev. D 74 (2006) 045025 [hep-ph/0602044]. 
[39] P. Kovtun and A. Starinets, Phys. Rev. Lett. 96 (2006) 131601 [hep-th/0602059].

[40] R. C. Myers, A. O. Starinets and R. M. Thomson, arXiv:0706.0162 [hep-th].

[41] P. Petreczky and D. Teaney, Phys. Rev. D 73 (2006) 014508 [hep-ph/0507318].

[42] F. Karsch and H. W. Wyld, Phys. Rev. D 35 (1987) 2518.

[43] A. Nakamura and S. Sakai, Phys. Rev. Lett. 94, 072305 (2005) [hep-lat/0406009].

[44] S. Gupta, Phys. Lett. B 597, 57 (2004) [hep-lat/0301006].

[45] G. Aarts, C. Allton, J. Foley, S. Hands and S. Kim, Phys. Rev. Lett. 99 (2007) 022002 [hep-lat/0703008].

[46] H. B. Meyer, arXiv:0704.1801 [hep-lat].

[47] M. Asakawa, T. Hatsuda and Y. Nakahara, Prog. Part. Nucl. Phys. 46, 459 (2001) [hep-lat/0011040].

[48] R.K. Bryan, Eur. Biophys. J. 18 (1990) 165.

[49] G. Aarts, C. Allton, J. Foley, S. Hands and S. Kim, Nucl. Phys. A 785 (2007) 202 [hep-lat/0607012].

[50] M. Asakawa and T. Hatsuda, Phys. Rev. Lett. 92, 012001 (2004) [hep-lat/0308034].

[51] S. Datta, F. Karsch, P. Petreczky and I. Wetzorke, Phys. Rev. D 69, 094507 (2004) [hep-lat/0312037].

[52] T. Umeda, K. Nomura and H. Matsufuru, Eur. Phys. J. C 39S1, 9 (2005) [hep-lat/0211003].

[53] A. Jakovac, P. Petreczky, K. Petrov and A. Velytsky, Phys. Rev. D 75 (2007) 014506 [hep-lat/0611017].

[54] T. Hatsuda, PoS LAT2006 (2006) 010.

[55] R. Morrin, A. O. Cais, M. Peardon, S. M. Ryan and J. I. Skullerud, Phys. Rev. D 74 (2006) 014505 [hep-lat/0604021].

[56] G. Aarts, C. Allton, M. B. Oktay, M. Peardon and J. I. Skullerud, arXiv:0705.2198 [hep-lat].

[57] F. Karsch, E. Laermann, P. Petreczky and S. Stickan, Phys. Rev. D 68 (2003) 014504 [hep-lat/0303017].

[58] G. Aarts and J. Foley [UKQCD Collaboration], JHEP 0702, 062 (2007) [hep-lat/0612007].

[59] T. Umeda, Phys. Rev. D 75 (2007) 094502 [hep-lat/0701005].

[60] A. Mocsy and P. Petreczky, Phys. Rev. D 73 (2006) 074007 [hep-ph/0512156].

[61] A. Mocsy and P. Petreczky, arXiv:0705.2559 [hep-ph].

[62] A. Mocsy and P. Petreczky, arXiv:0706.2183 [hep-ph].

[63] M. Laine, O. Philipsen, P. Romatschke and M. Tassler, JHEP 0703 (2007) 054 [hep-ph/0611300].

[64] M. Laine, JHEP 0705 (2007) 028 [arXiv:0704.1720 [hep-ph]]. 